\title{
mesure de l'affouillement maximum dû à une crue par diagraphie radio-activité naturelle après la crue
}

\author{
in situ determination of the maximum depth \\ of scour using natural radio activity measurements \\ after the flood
}

C. MIEUSSENS

Laboratoire Régional

des Ponts et Chaussées de Toulouse *

\section{Résumé}

La connaissance de l'affouillement maximum en rivière est une donnée indispensable pour le choix d'une cote de fondation ou pour le dimensionnement de protections.

Le projeteur utilise généralement des formules empiriques basées sur des observations ou bien issues d'expérimentations sur modèles réduits. Mais l'information la plus utile est la mesure de l'affouillement maximum pendant la crue, les relevés après la crue n'étant pas représentatifs à cause de la sédimentation lors de la décrue.

On décrit dans cet article une méthode originale expérimentée en vraie grandeur sur la Garonne, basée sur la mesure de diagraphie radio-activité naturelle. Elle permet après la crue de déterminer l'interface entre le matériau non affouillé et les matériaux sédimentés dans un tube de mesure placé au centre d'un puits oủ le matériau naturel a été remplacé par un matériau similaire mais de radioactivité naturelle différente.

\section{Abstract}

The prediction of the maximum depth of scour is fundamental to the design of underwater foundations. This depth governs the choice of the foundation level and/or the design of protecting works.

At the present time, the engineer in charge of the design generally uses empirical or semi-empirical formula, which are based on in situ observations or laboratory models. However, the most useful information comes the direct measurement of the maximum depth of erosion during an actual flood; measurements made after the flood are generally not representative because of the sediments which refill the eroded river bed as the river level goes down.

This paper deals with an original method of measuring the maximum depth of scour, which was tried on the Garonne river. This method is based on the measurement of natural radio activity. These measurements allow the interface between the non eroded material and the sediment to be determined. The measurements are made through a vertical tube placed in the middle of a pit which is dug prior to the flood and filled with material that has a radio activity different from the surrounding natural soil.

\footnotetext{
- 1, avenue du Colonel-Roche, Complexe aérospatial, 31400 Toulouse.
} 
La connaissance de l'affouillement maximum en rivière, affouillement général pour l'ensemble du lit ou local au voisinage d'un ouvrage, est une donnée indispensable, tant pour l'étude d'un nouveau projet que pour le dimensionnement des protections qui assureront la pérennité d'un ouvrage existant, menacé par l'évolution du fond du lit.

Le projecteur dispose généralement de formules permettant d'évaluer l'affouillement maximum en fonction des données hydrauliques, géotechniques et géométriques. La plupart de ces formules sont issues d'expérimentations sur modèles réduits et quelquefois sur des synthèses d'observations sur ouvrages réels. Pour l'affouillement local autour des piles en milieu pulvérulent on pourra se reporter à la publication de BREUSERS et al. (1977) qui dresse l'état des connaissances. Très souvent les études ont porté sur les sédiments pulvérulents fins $(<1 \mathrm{~mm})$ mais on manque d'observations et de données sur les sols grossiers et surtout sur les sols cohérents.

Les formules proposées dans la littérature ne sont donc pas suffisantes, il convient donc de s'appuyer sur les résultats d'observations, mais on se heurte alors à une autre difficulté qui est la mesure de l'affouillement maximum pendant la crue, les relevés après la crue n'étant pas représentatifs à cause de la sédimendation du fond mobile lors de la décrue.

Nous rappellerons d'abord les principes d'un certain nombre de procédés qui ont été imaginés et testés puis nous décrirons une méthode originale expérimentée en vraie grandeur sur la Garonne pendant la période 1980-1984, basée sur une mesure de diagraphie «radio-activité naturelle ( $R . A . N$.) qui permet après la crue de déterminer la position de l'interface entre le matériau non affouillé et les matériaux sédimentés, le tube de mesure étant placé au centre d'un puits dans lequel le matériau naturel de la rivière a été remplacé par un sol de granulométrie similaire, mais de R.A.N. différente.

\section{PRINCIPALES TECHNIQUES DE MESURES DE L'AFFOUILLEMENT MAXIMUM}

Parmi les méthodes et les appareillages permettant la mesure de l'affouillement maximum on peut citer les suivantes :

L'écho-sondeur : selon le principe du sonar, l'échosondeur permet de mesurer le temps séparant l'émission d'une onde haute fréquence $(200 \mathrm{~Hz})$ de sa réception après réflexion sur le fond du lit, qui est donc directement fonction du tirant d'eau au point considéré. Cet appareillage donne des résultats très satisfaisants en bathymétrie et son utilisation pour des mesures d'affouillements à proximité des appuis des ouvrages est très séduisante à cause du faible coût de sa mise en ceuvre. Toutefois un certain nombre de problèmes doivent être résolus pour rendre la mesure fiable et précise :

- l'absorption des ondes par les matériaux en suspension,

- les réflexions parasites sur l'ouvrage,
- la difficulté d'avoir une information suffisamment ponctuelle, les fosses au voisinage des appuis pouvant être très étroites.

La mise au point de ce système avec enregistrement et déclanchement automatique à partir d'un certain niveau de crue fait actuellement l'objet d'une étude au Laboratoire Régional des Ponts et Chaussées de Bordeaux.

Mesure par résistivité électrique : l'eau ayant une résistivité très différente de celle du matériau, la position du fond du lit peut être détectée en mesurant la résistance entre des électrodes déposées à des profondeurs croissantes. Cette technique a l'avantage de permettre un enregistrement continu, cependant elle nécessite probablement un entretien périodique.

Citée par S.H. KÜHN et al. (1961), cette méthode a fait l'objet d'une expérimentation récente en vraie grandeur par le Laboratoire Régional des Ponts et Chaussées de Strasbourg.

Méthodes optiques : le principe consiste à observer directement, avec un miroir descendu dans un tubage comportant des fenêtres, le niveau du fond du lit pendant la crue. Cette technique a été utilisée sur le grand canal de Banlève à Toulouse.

Essais mécaniques : les essais de pénétration statique ou dynamique ou bien l'exploration par les méthodes géophysiques permettent de distinguer les horizons peu compacts des horizons plus compacts donc a priori n'ayant pas subi un affouillement.

Diagraphie nucléaire $\gamma$ : la mesure de diagraphie nucléaire par gammadensimétrie est également un moyen d'apprécier les variations de densité des matériaux alluvionnaires et ainsi de distinguer les sédiments en place de ceux déposés après une crue. Cette méthode est citée par APPE et NICOLLET (1973) qui font des réserves sur la précision des mesures. Sur la base d'expériences en vraie grandeur S.N. KUHN et A.A.B. WILLIAMS (1961) indiquent que la seule mesure de densité n'est pas une information suffisante pour en déduire un affouillement maximum.

\section{PRINCIPE DE LA MESURE DE L'AFFOUILLEMENT MAXIMUM PAR LA DÉTERMINATION DE L'INTERFACE DU MATÉRIAU SÉDIMENTÉ APRÊS LA CRUE AVEC UN MATÉRIAU DE SUBSTITUTION DE R.A.N. DIFFÉRENTE}

Le principe de la méthode expérimentée en vraie grandeur à Bourret sur la Garonne est décrit sur la figure 1.

- Le croquis 0 donne la configuration du site à instrumenter au voisinage d'une pile en rivière. Les alluvions affouillables sont supposées être par exemple à prédominance granitique, symbolisées par $(+)$ et par conséquent caractérisées par une forte radio-activité naturelle R.A.N. 


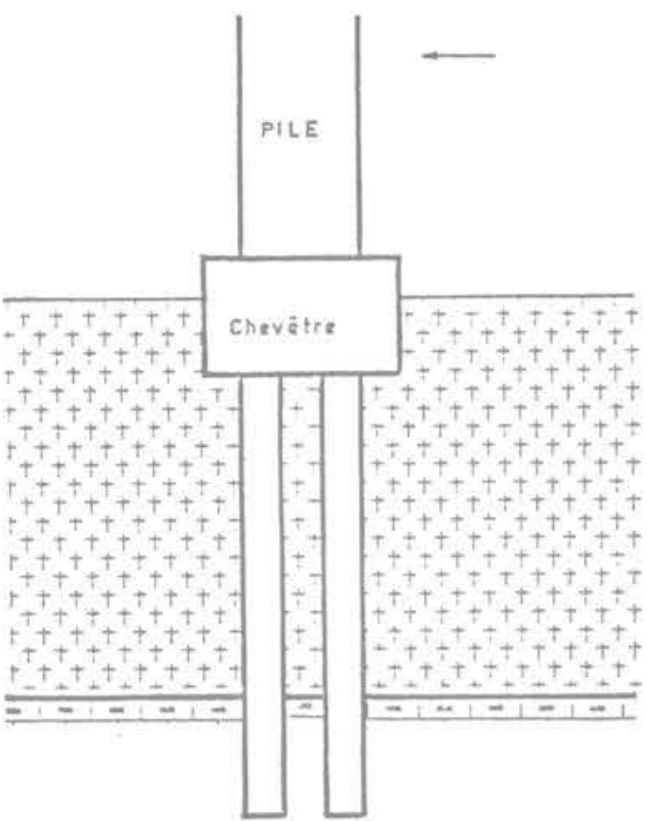

(0) Configuration du site 0 instrumenter.

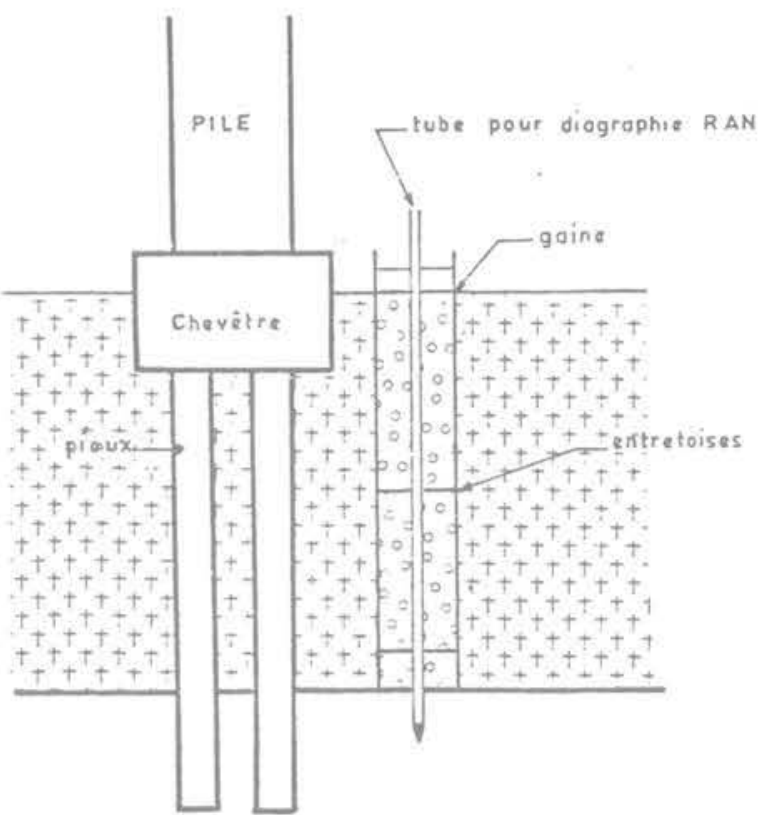

(1) Phase 1 de l'instrumentation Constitution a linterieur d'une gaine, d'un puits de matériauo Ude RAN très differrente du matériau ${ }_{+}^{+}+$de la rivière et mise en place du tube de diagraphie RAN

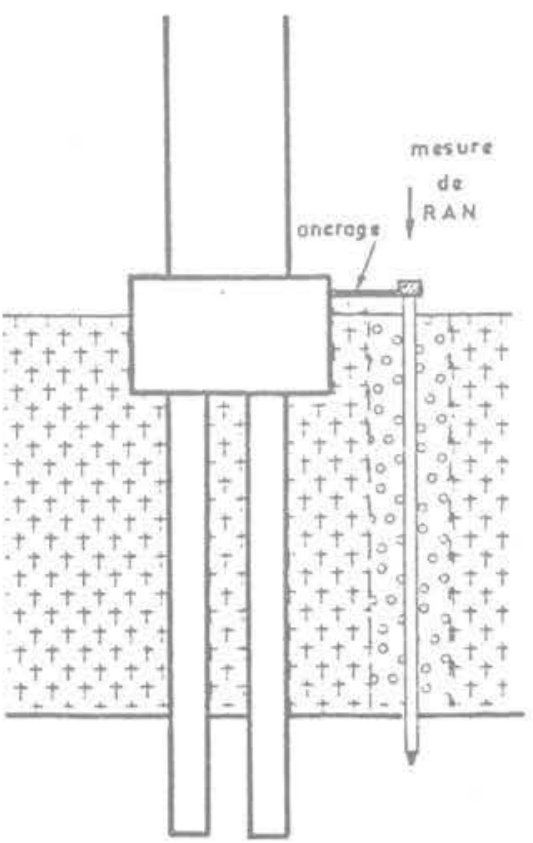

(2) Phase 2 de l'instrumentation la gaine est retirée et le tube de diagraphie scallé en tète mesure de zero de $R A N$

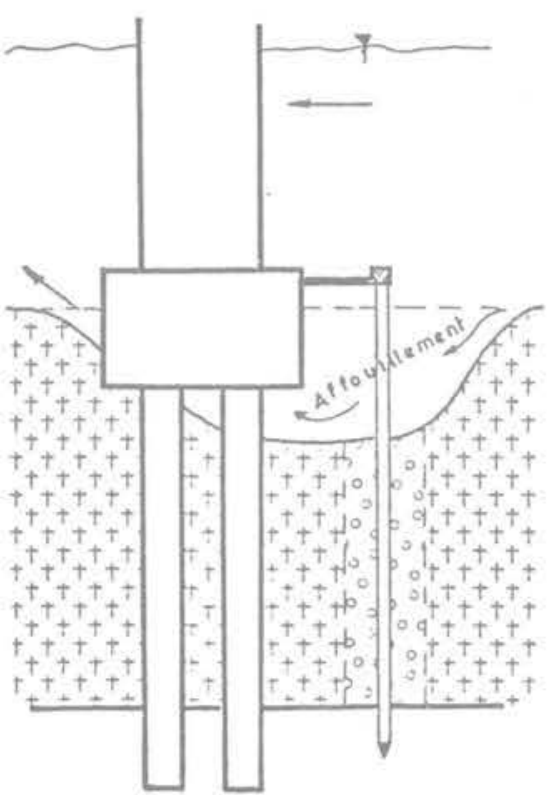
(3) Affouillement maximum
pendant ia crue

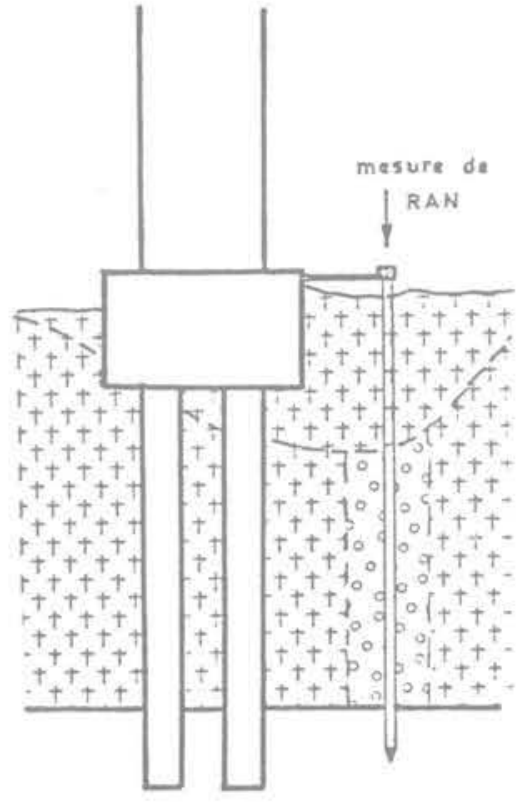

(4) Détermination par mesure de RAN de l'interface entre les matériaux ${ }^{+}+{ }^{+}+e t_{0}^{\circ}$,

Fig. 1. - Principe de la méthode de l'affouillement maximum par R.A.N. après la crue. 
- Aussi prêt que possible du chevêtre, on réalise un forage tubé en gros diamètre D, au moyen par exemple d'une sondeuse à benne. Le tubage de travail est équipé d'entretoises pour centrer le tube de diagraphie de diamètre $\mathrm{d}$; le tube de diagraphie est éventuellement fiché par battage dans le substratum compact. Le tube de travail est alors rempli avec un matériau symbolisé par (o) de R.A.N. très différente (un calcaire dans l'exemple choisi ici) mais de même granulométrie que les alluvions naturelles. Le tubage de travail est retiré au fur et à mesure du remplissage avec le matériau de substitution.

- Le croquis 2 précise la configuration du site instrumenté. Il y a intérêt à rendre la tête du tube de diagraphie solidaire du chevêtre.

Une mesure de "zéro * de R.A.N. est alors faite dans cette configuration.

- Le croquis 3 décrit le phénomène d'affouillement pendant le maximum de la crue.

- Après la crue, le matériau charrié par la rivière peut remplir partiellement, totalement ou même plus haut qu'initialement le cône d'affouillement local comme le montre le croquis 4.

Le niveau de l'affouillement maximum correspond à l'interface calcaire/matériau naturel resédimenté. Il est déterminé par un nouveau profil de R.A.N. que l'on comparera à la mesure de zéro.

\section{Caractéristiques du matériel de R.A.N. :}

Le matériel de R.A.N. fait partie d'un ensemble de diagraphie conçu et réalisé par les laboratoires des Ponts et Chaussées. Il a été imaginé pour permettre le repérage de niveaux géologiques et en particulier l'établissement de corrélations stratigraphiques. La différenciation des couches se fait en fonction des variations de teneurs en éléments radio-actifs.

Les mesures se font dans un forage tubé ou non (tubage plastique ou métallique) avec ou sans eau. Le diamètre doit être compris entre 35 et $150 \mathrm{~mm}$.

La sonde a un diamètre de $27 \mathrm{~mm}$ et une longueur de $650 \mathrm{~mm}$. Elle est mise en œuvre au moyen d'un treuil, la vitesse de déroulement $(0,5$ à $5 \mathrm{~cm} / \mathrm{s})$. L'enregistrement graphique se fait sur un papier dont le déroulement est asservi à celui du câble.

\section{EXPÉRIMENTATION POUR LA MISE AU POINT DE LA MÉTHODE}

Le principe de la méthode une fois défini, il restait encore à faire une expérimentation pour préciser les conditions de mise en œuvre et en particulier choisir le diamètre minimum $\mathrm{D}$ du forage rempli par le matériau de substitution pour détection suffisamment aisée de l'interface.

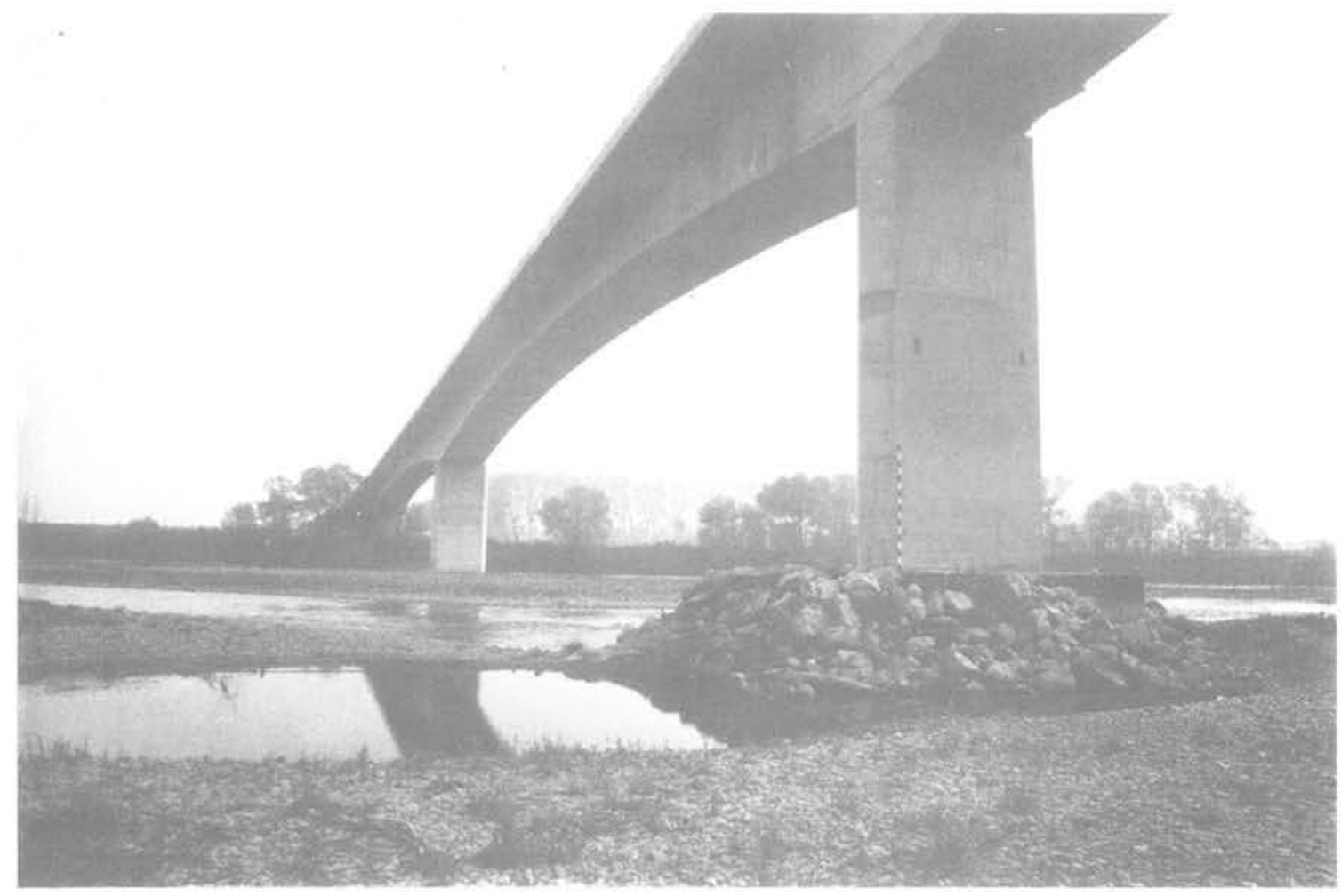

Fig. 2. - Vue partielle de l'ouvrage terminé :

viaduc de franchissement de la Garonne à Bourret

(au premier plan : pile instrumentée). 
A cet effet une expérience en vraie grandeur a été réalisée à proximité d'une des piles du nouveau viaduc qui franchit la Garonne à Bourret dans le Tarn-etGaronne.

La photo de la figure 2 donne une vue partielle du viaduc terminé. Au premier plan de cette photo on peut voir la pile en rive gauche qui a fait l'objet de l'instrumentation décrite. La reconnaissance des sols pour l'étude de l'ouvrage avait mis en évidence pour cet appui une épaisseur de 4 à $5 \mathrm{~m}$ de graves au-dessus du substratum marneux. La figure 3 donne la courbe granulométrique de ces graves ainsi que celle du matériau calcaire de substitution.

Les alluvions graveleuses comportent une assez grande variété de minéraux, mais la prédominance des gneiss et des granites leur confère une radio-activité naturelle assez grande.

On peut observer sur la figure 2 que le chevêtre est anormalement haut au-dessus du fond du lit. Cette situation est la conséquence de la crue de mai 1977 $\left(\mathrm{Q}-2500 \mathrm{~m}^{3} / \mathrm{s}\right.$ - fréquence de retour 10 ans) qui, pendant les travaux et du fait des installations de chantiers, avait entraîné les alluvions sur une grande épaisseur. La berge en rive gauche a ainsi été déplacée de façon irréversible de quelques dizaines de mètres, avec pour conséquence de transformer un des appuis sur berge en appui en rivière.

En outre les calculs montraient que pour des crues de fréquences de retour de 1 à 2 ans l'affouillement local devait être significatif. Le site paraissait donc favorable à une instrumentation.
Précisons que vis-à-vis de la sécurité de l'ouvrage l'hypothèse d'un affouillement local total avait été retenue, le dimensionnement des pieux et leur protection étant adaptés à cette hypothèse.

\subsection{Simulation d'un affouillement}

La première phase de l'expérimentation a consisté à simuler un affouillement de l'ordre de $1 \mathrm{~m}$ suivie par une sédimentation de $1 \mathrm{~m}$ également autour des forages de diamètre $D$ différents : $0,4-0,6-0,8$ et $1 \mathrm{~m}$.

Cette simulation a été réalisée au droit des quatre tubes de diagraphie disposés à l'aval de la pile et dont l'implantation est précisée sur les figures 4 et 6 .

La figure 4 explicite la procédure suivie pour faire cette simulation :

- Réalisation d'une fouille à la pelle mécanique selon le profil indiqué sur la figure;

- Mise en place des 4 tubes de travail de diamètre D;

- Battage des tubes de diagraphie au centre des tubes de travail ;

- Remplissage simultané de la fouille avec les alluvions de la Garonne et de l'intérieur des tubes avec du calcaire. Les tubes de travail sont retirés au fur et à mesure.

- Après l'achèvement des quatre colonnes de matériau calcaire le remblaiement avec le matériau naturel de la Garonne est poursuivi autour des tubes de diagraphie sur une épaisseur de 0,8 à $0,95 \mathrm{~m}$.

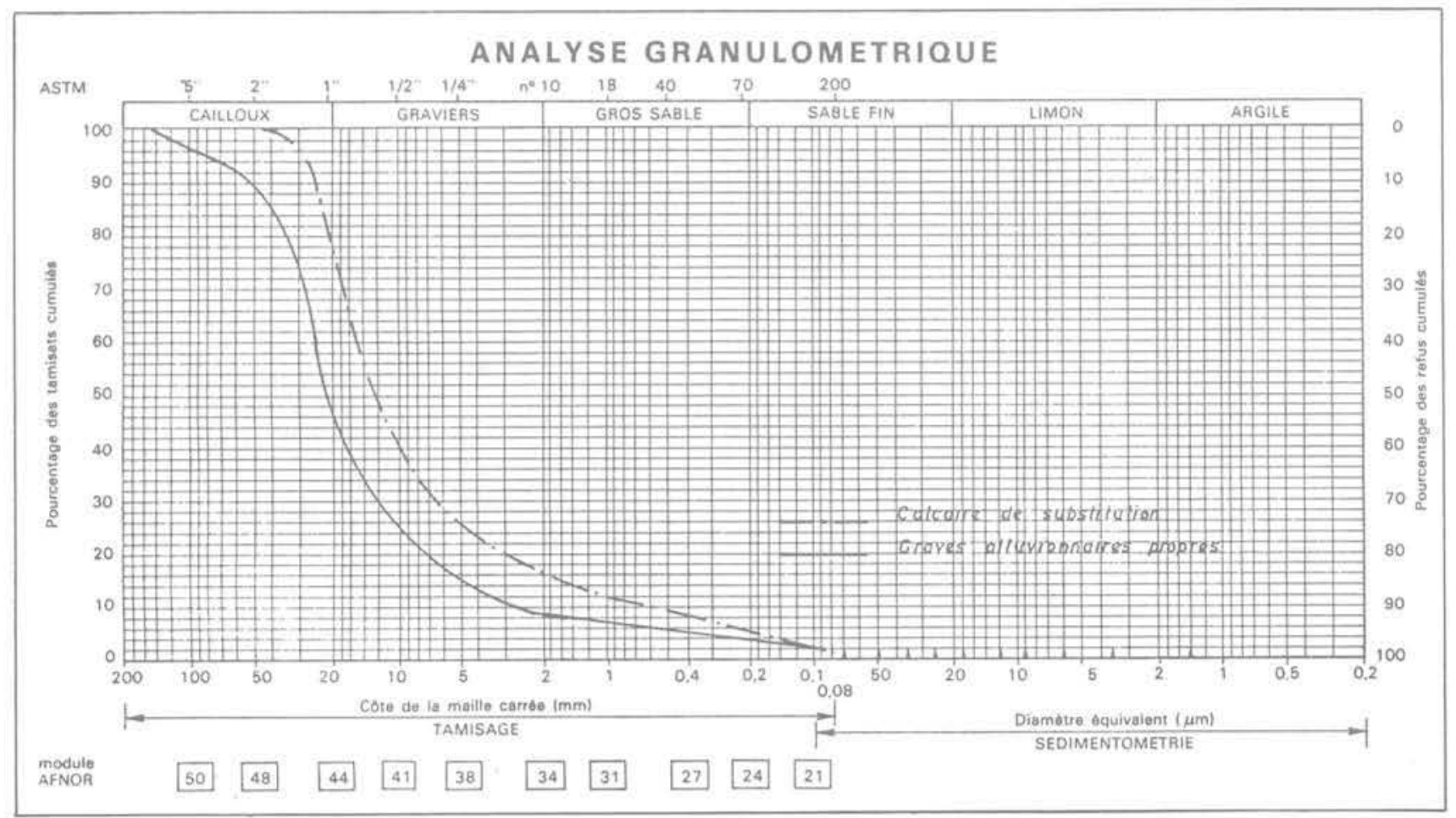

Fig. 3. - Analyse granulométriques des matériaux alluvionnaires nature/s et du calcaire de substitution. 


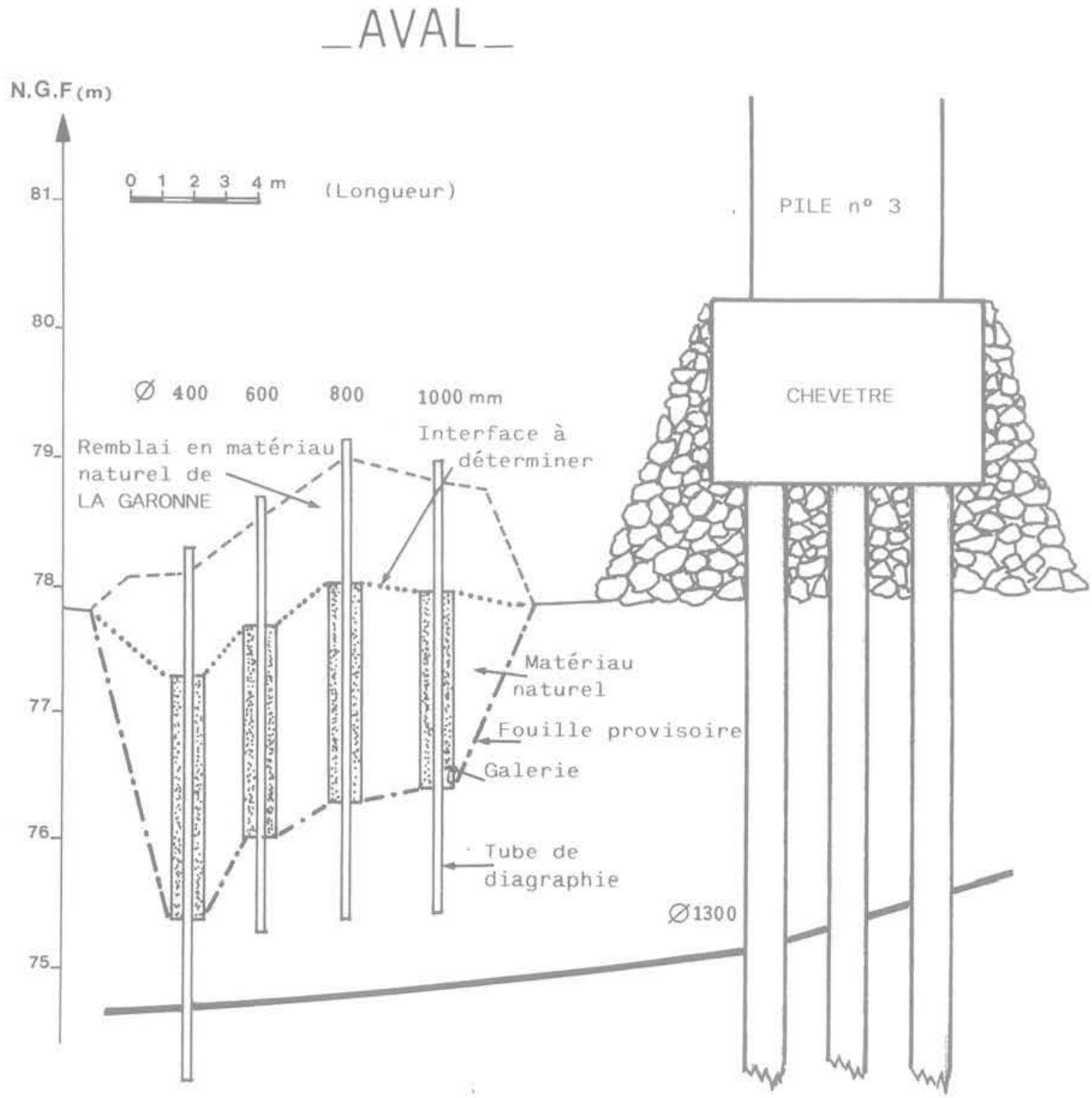

Fig. 4. - Instrumentation à l'aval pour la simulation d'un affouillement de $1 \mathrm{~m}$ environ.

La simulation de la mesure d'un affouillement de l'ordre de $1 \mathrm{~m}$, suivi d'une sédimentation identique consiste donc à retrouver par diagraphie R.A.N. la position de l'interface définie sur la figure 4.

Les résultats pour les quatre tubes de diagraphie sont donnés sur la figure 5. La plage de transition a une largeur variant de 0,36 à $0,48 \mathrm{~m}$ et le milieu de cette plage correspond à quelques centimètres près à la position réelle de l'interface. On peut noter aussi que le contraste de comptage de R.A.N. entre le matériau de substitution et le matériau sédimenté est net à partir de $\mathrm{D}=0,6 \mathrm{~m}$, mais encore satisfaisant pour $\mathrm{D}=0,4 \mathrm{~m}$.

Une expérience similaire a été faite avec les tubes de diagraphie disposés à l'amont de l'ouvrage pour un affouillement simulé de $0,4 \mathrm{~m}$. Les résultats sont tout à fait comparables.

\subsection{Mesures d'affouillement réels} (de 1979 à 1984)

Après achèvement des expériences de simulation décrites dans le paragraphe 3.1., le site a été aménagé conformément au profil en long de la figure 6 . Cette figure précise le niveau du fond du lit à différentes dates et les cotes de l'affouillement maximum déduites des mesures de R.A.N.

A l'amont on a reporté les résultats de trois séries de mesures après affouillement.

$N^{\circ} 1$ - Septembre 1980. - L'affouillement correspond à la courbe $1 \mathrm{~b}$ alors que le fond du lit $1 \mathrm{a}$ est au-dessus du niveau initial 0 . 

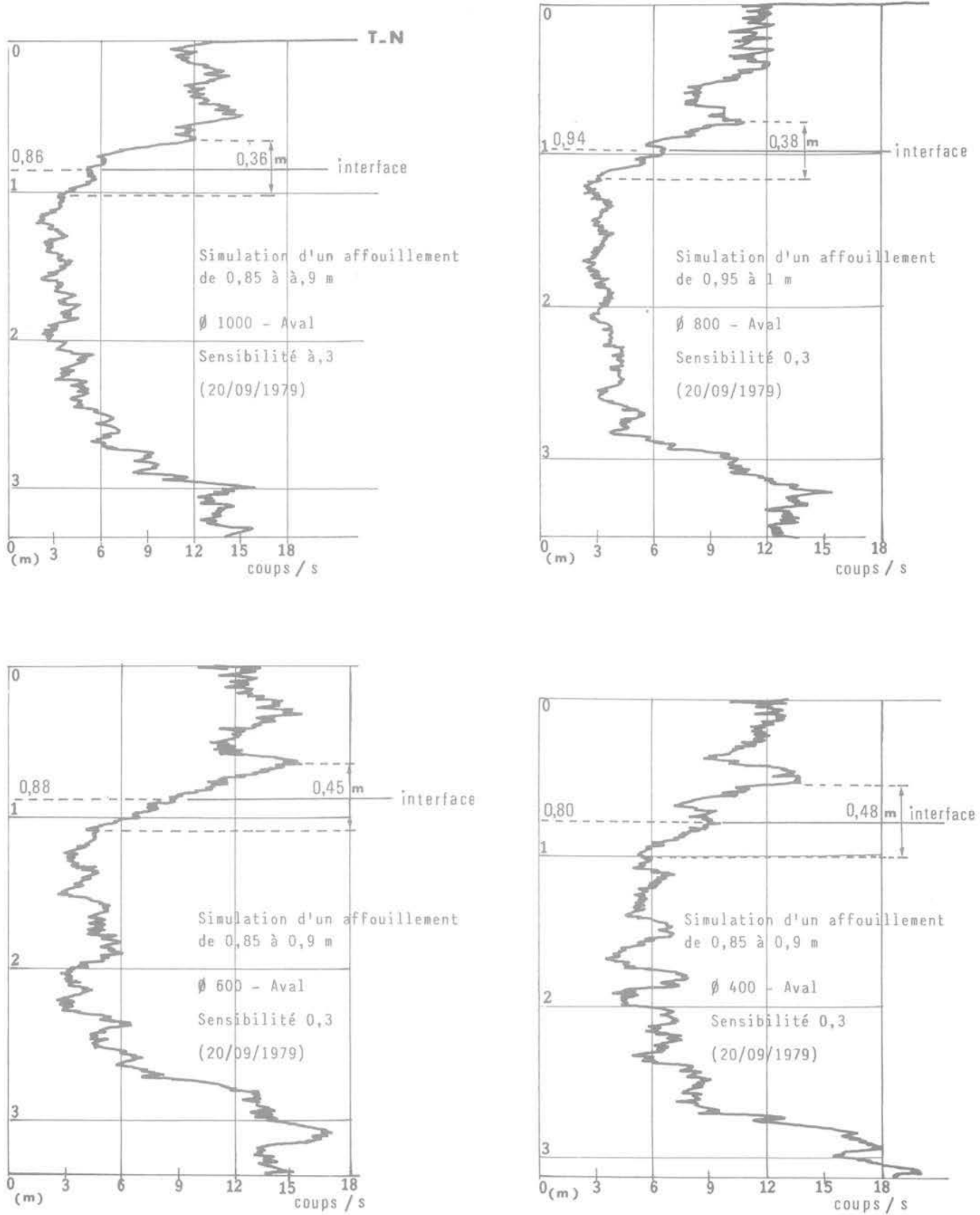

Fig. 5. - Simulation d'un affouillement de $1 \mathrm{~m}$ environ suivi d'une sédimentation de même amplitude. Résultats des mesures de R.A.N. pour quatre valeurs de D. 


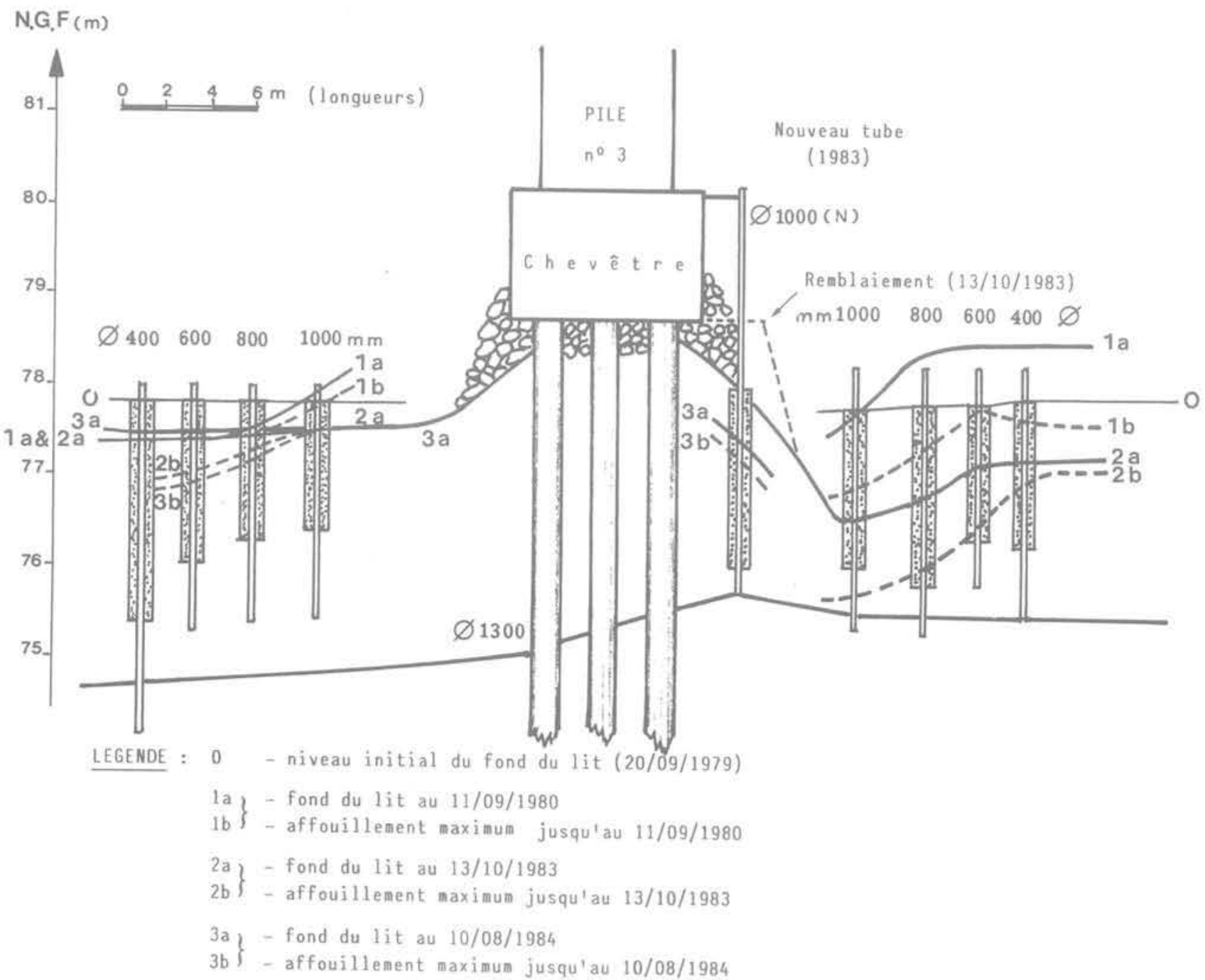

Fig. 6. - Profil en long de l'instrumentation réalisée.

Evolution du fond du lit

et de la cote de l'affouillement maximum.

$N^{\circ} 2$ - Octobre 1983. - On observe une fosse d'affouillement 2 a mais l'affouillement maximum a été relativement plus important $2 b$. A cette date un nouveau tube a été placé à proximité du chevetre $(\varnothing 1000 \mathrm{~N}$ ), après qu'une partie des enrochements ait été enlevée.

$N^{\circ} 3$ - Août 1984. - Les mesures ne sont possibles en amont que dans le nouveau tube près du chevêtre.

A l'aval, pour les mêmes périodes, l'évolution est moins importante tant pour les variations de la cote du fond du lit que pour celle de l'affouillement "pendant la crue».
La figure 7 donne quelques exemples de mesures de diagraphie R.A.N. avant et après une crue (crue de janvier 1980). Malgré un changement d'échelle de comptage (dû à un changement de matériel), ces graphiques confirment que la méthode proposée permet une détermination assez précise de l'interface entre le niveau affouillé et le matériau sédimenté après la crue.

\section{CONCLUSIONS}

L'expérimentation décrite avait pour objectif de mettre en évidence la faisabilité de la méthode, elle n'aborde donc pas toutes les difficultés que l'on pourrait rencon- 
MESURE DE ZERO

20. 09.79

NG F

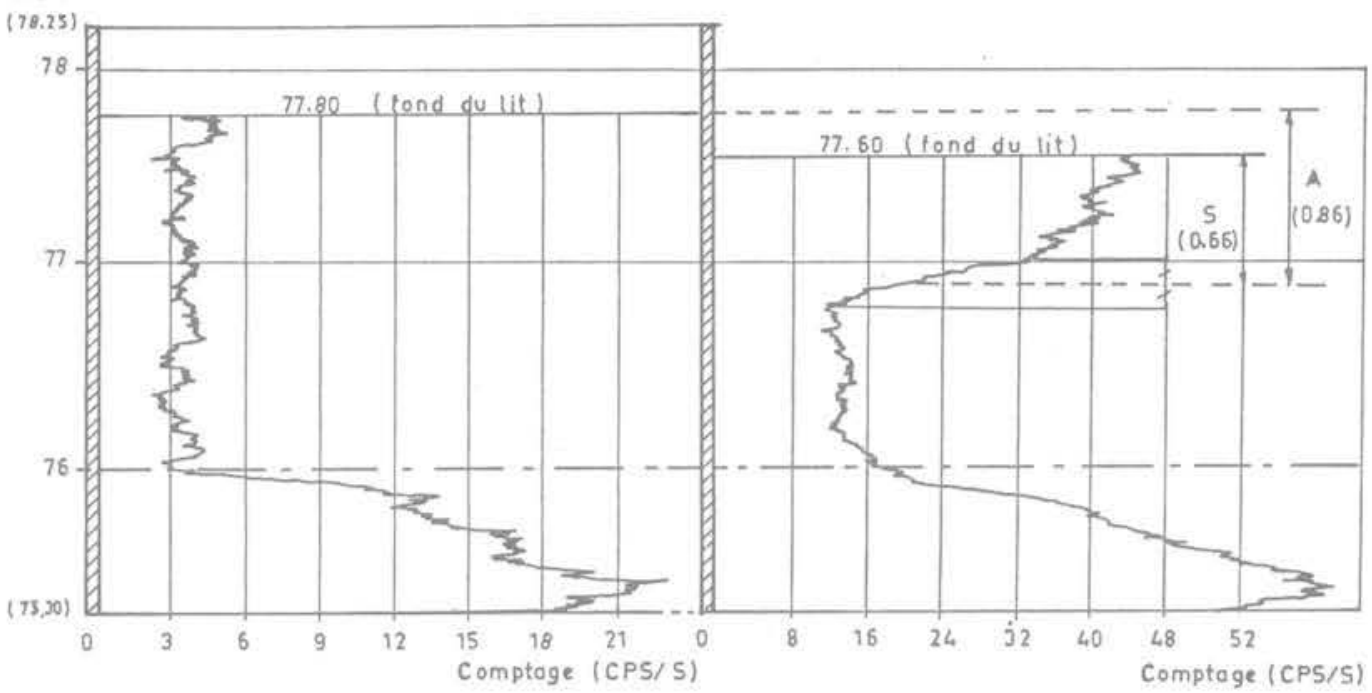

MESURE DE ZERO

20 - $09-79$

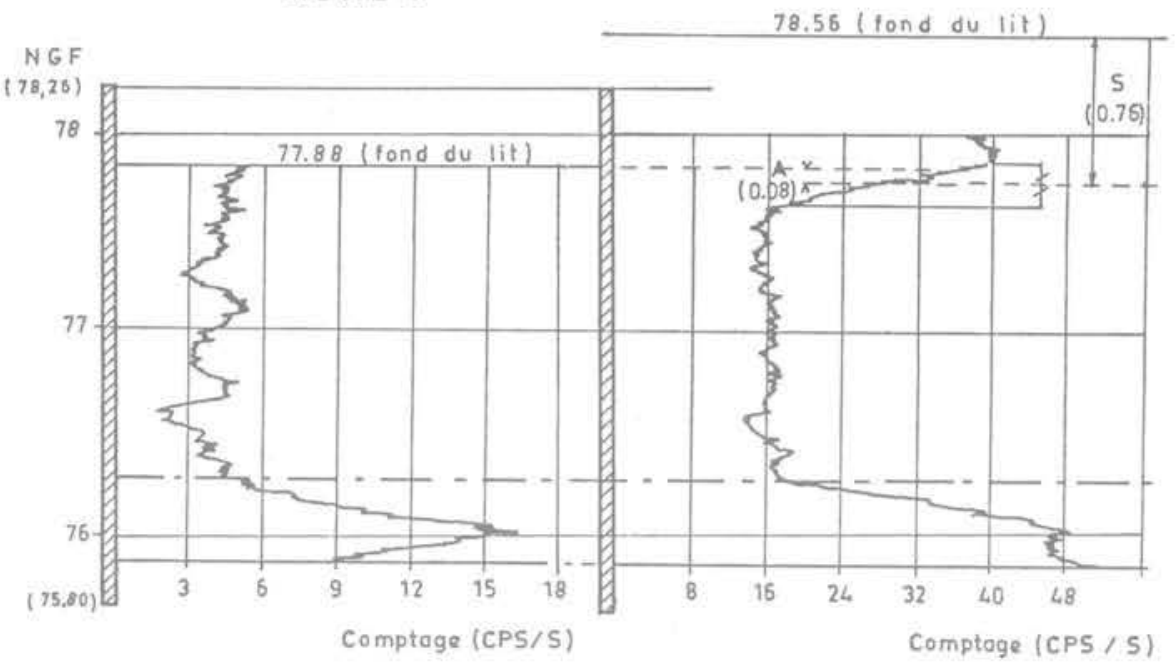

MESURE DE ZERO

20.09 .79

MESURE DU 11.09 .80

NGF

$$
78
$$

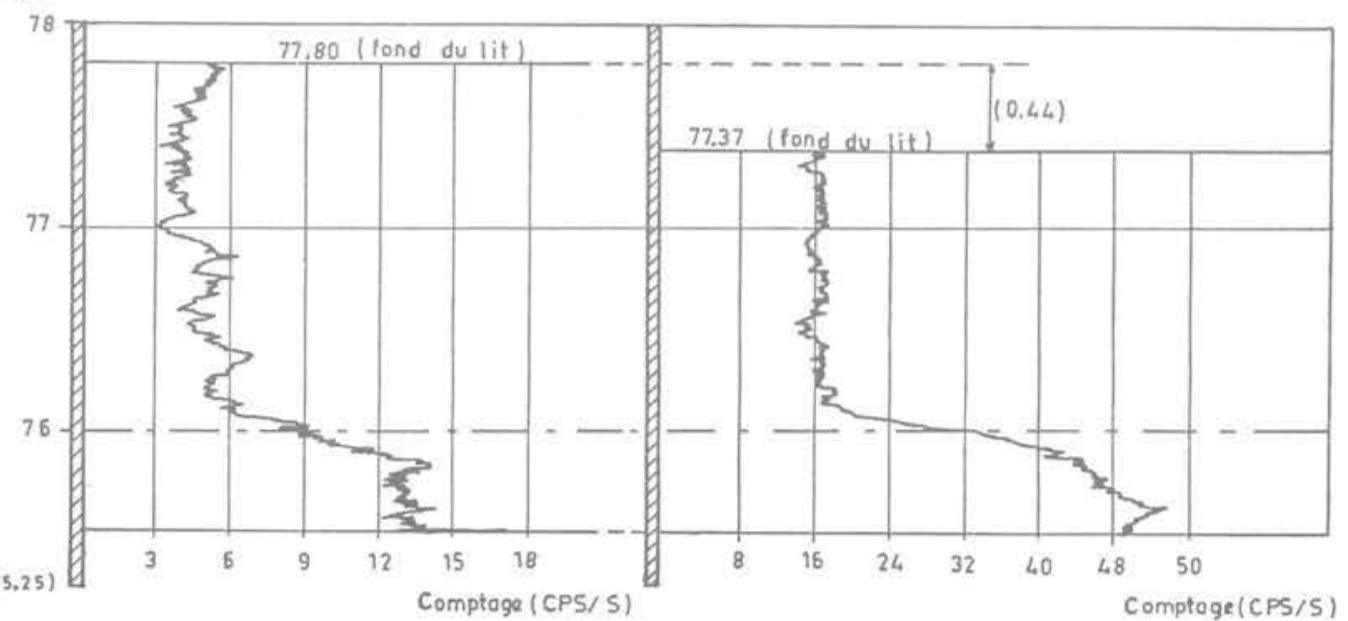

Fig. 7. - Exemples de mesures de diagraphies R.A.N. avant et après une crue. 
trer et qui évidemment seront variables d'un site à l'autre. On peut cependant attirer l'attention sur les points suivants :

- La méthode n'est envisageable que si on peut trouver un matériau de substitution de caractéristiques R.A.N. nettement différentes de celles des alluvions en place. On a vérifié ici que les résultats sont satisfaisants pour un rapport de comptage de 1 à 3 entre les deux matériaux.

- Le diamètre D doit être suffisant pour que le matériau naturel environnant n'influence pas trop le comptage R.A.N. dans le matériau de substitution. Cette condition était satisfaite ici à partir de $\mathrm{D} \geqslant 0,6 \mathrm{~m}$, mais les mesures avec $D=0,4$ sont encore assez précises, il semble toutefois que ce soit une limite inférieure à ne pas dépasser.

- Le matériel nécessaire pour la mise en ceuvre du tube de travail sera adapté aux conditions d'accès et à la profondeur de l'instrumentation. D'une façon générale une sondeuse à benne avec un tubage de diamètre suffisant devrait donner des résultats satisfaisants.

- Notre expérience a montré que la principale cause de détérioration des tubes résulte des impacts de corps flottants. Ainsi la plupart des tubes arasés assez près du fond du lit ont bien résisté pendant cinq ans aux différentes crues de la Garonne. Par contre le dernier tube rendu solidaire du chevêtre a été tordu par des corps flottants (la mesure n'a été possible qu'après l'avoir scié à la base).

- Il est important de bien étancher les têtes des tubes de mesure R.A.N. Dans le cas contraire la sédimentation des fines apportées par les crues risque de les boucher progressivement. Ainsi, en cinq ans, les tubes placés sur le site de Bourret se sont remplis de 0,5 à 1,2 m de sédiments.
Seules d'autres applications sur d'autres sites permettront de mieux préciser la condition optimale de mise en cuvre. Mais le principe de faisabilité nous semble suffisamment établi pour que la méthode soit utilisée à des fins de surveillance et aussi de recherche pour une meilleure connaissance des phénomènes.

\section{BIBLIOGRAPHIE}

1. H.W. SHEN, V.R. SCHNEIDER and S. KARAKI, Local scour around bridge piers, J. of the Hydraulics Division. Proc ASCE, H.Y. 6 (Nov. 1969).

2. N.N.C. BREUSERS, G. NICOLLET et H.W. SHEN, Local scour around cylindrical piers, J. of Hydraulic Research 15 (1977), n 3 .

3. H. APPE et G. NICOLLET, Mesure des affouillements in situ au pied des piles de pont, Note à diffusion restreinte C 43/73/50 du Laboratoire National d'Hydraulique de Châtou (1973).

4. G. NICOLLET, Mesure des affouillements in situ par un écho-sondeur, Note à diffusion restreinte C 43/74/66 du Laboratoire National d'Hydraulique de Châtou (1974)

5. S.H. KÜHN et A.A.B. WILLIAMS, Scour pits and soils profiles determination in river beds, Communication au Ve Congrès I.M.S.T.F. (Paris, 1961). Vol. 1, 2/11, pp. 487-490 